Supplement of Geosci. Model Dev., 11, 4489-4513, 2018

https://doi.org/10.5194/gmd-11-4489-2018-supplement

(C) Author(s) 2018. This work is distributed under

the Creative Commons Attribution 4.0 License.

(c) (1)

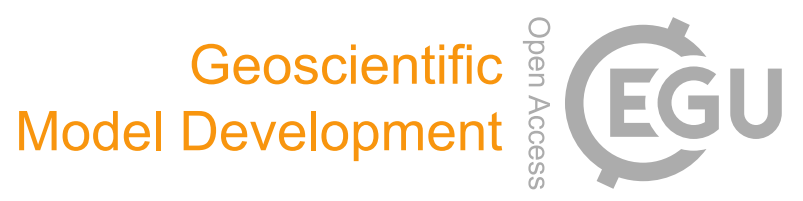

Supplement of

\title{
On the impact of recent developments of the LMDz atmospheric general circulation model on the simulation of $\mathrm{CO}_{2}$ transport
}

M. Remaud et al.

Correspondence to: Marine Remaud (mremaud@lsce.ipsl.fr)

The copyright of individual parts of the supplement might differ from the CC BY 4.0 License. 


\section{Sensitivity to the surface fluxes: Description of the experiments}

In order to test the robustness of the main findings to the prescribed $\mathrm{CO}_{2}$ fluxes, we performed two additional simulations with, respectively, physics 5A and 6A driven by the 3 hourly CarbonTracker CT2017 (CT) $\mathrm{CO}_{2}$ fluxes interpolated at horizontal resolution $3.75^{\circ} \times 1.90^{\circ}$ (https://www.esrl.noaa.gov/gmd/ccgg/carbontracker/). The CT2017 fluxes have been optimized using 5 transport model TM5 run at a global $3^{\circ} \times 2^{\circ}$ resolution with a nested regional grid over North America at $1^{\circ} \times 1^{\circ}$ resolution. Table 1 describes our model simulations considered in this supplement. Even though the two inversion systems that produced the CAMS and CT fluxes differ in terms of assimilation approaches, model transport and prior fluxes, they assimilate the same surface measurements to a large extent, so that the question of the lack of independence of our $\mathrm{CO}_{2}$ simulations to most surface measurements still remain. Figure 1 shows the mean differences between the CAMS and the CT fluxes at annual and seasonal scales. Regarding the seasonal average over the period 2005-2010, the noticeable differences are located over tropical and convective areas (Africa, Northern Australia and Brazil) and over Siberia in boreal winter. Those regions are relatively poorly constrained by the inverse systems.

\begin{tabular}{llcccc} 
Version & Physics & \multicolumn{2}{c}{ Horizontal resolution (longitude $\times$ latitude) } & Number of & $\mathrm{CO}_{2}$ fluxes \\
& & \multicolumn{1}{c}{ degrees } & $96 \times 95$ & 39 & CAMS 15r4 \\
\hline 5A-96L39 & 5A (old physics) + ORCHIDEE & $3.75^{\circ} \times 1.90^{\circ}$ & $96 \times 9$ levels & \\
6A-96L39 & 6A (new physics) + ORCHIDEE & $3.75^{\circ} \times 1.90^{\circ}$ & $96 \times 95$ & 39 & CAMS 15r4 \\
5A-96L39-CT & 5A (old physics) + ORCHIDEE & $3.75^{\circ} \times 1.90^{\circ}$ & $96 \times 95$ & 39 & CarbonTracker CT2017 \\
6A-96L39-CT & 6A (new physics) + ORCHIDEE & $3.75^{\circ} \times 1.90^{\circ}$ & $96 \times 95$ & 39 & CarbonTracker CT2017
\end{tabular}

Table 1. Description of the simulations.

\section{Sensitivity to the surface fluxes: Results}

Figure 2 shows the impacts of the surface fluxes and of the parameterization assumptions on the zonal-mean mixing ratio of $\mathrm{CO}_{2}$ over 2005-2010 boreal summers. The impact of the surface fluxes on the zonal mean structure of the $\mathrm{CO}_{2}$ values does 
a) annual (CT)

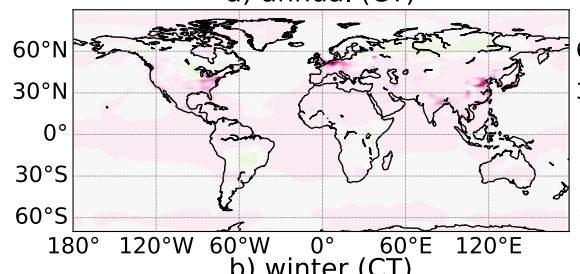

b) winter $(\mathrm{CT})$

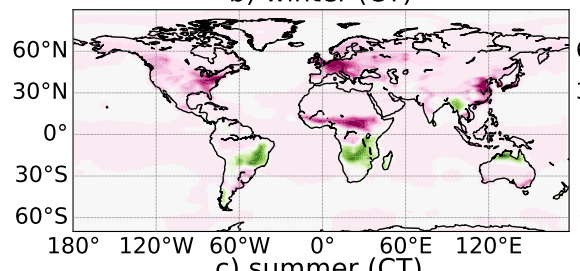

C) summer (CT)

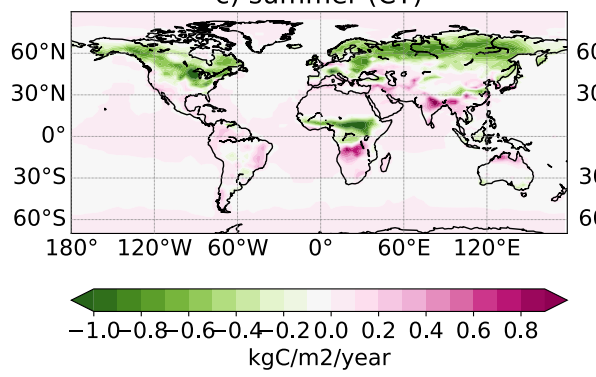

d) annual (CT-CAMS)

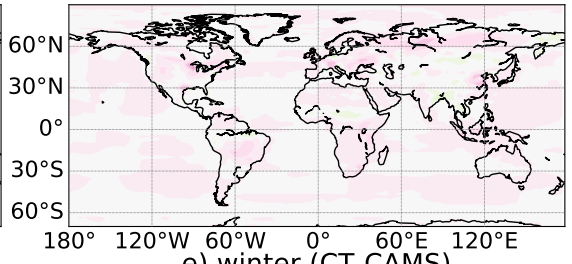

e) winter (CT-CAMS)

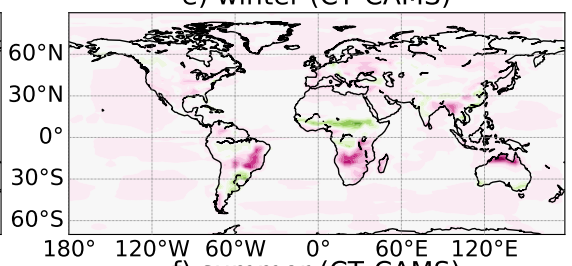

f) summer (CT-CAMS)

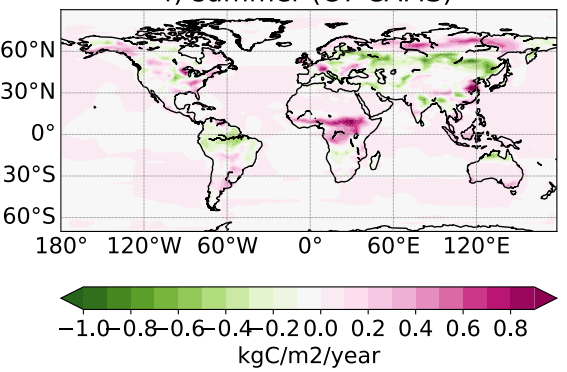

Figure 1. a-c) Map of the $\mathrm{CO}_{2}$ prescribed fluxes from CarbonTracker CT2017 (CT) averaged over 2005-2010 period (a), over 2005-2010 boreal winters (b) and 2005-2010 boreal summers (c). d-f) Same as a-c) but for the difference between CarbonTracker CT2017 (CT) and Copernicus $\mathrm{CO}_{2}$ fluxes (CAMS-15r4).

not exceed $1 \mathrm{ppm}$, with higher concentrations in the northern hemisphere related to stronger surface fluxes over Siberia. The use of a different surface flux dataset does not change the effect of the physics on the zonal mean mixing ratio of $\mathrm{CO}_{2}$. The negative anomalies are still more trapped within the boundary layer because of the convective inhibition, resulting in a positive anomaly in the mid troposphere and a negative anomaly at $1 \mathrm{~km}$.

Figure 3 depicts the sensitivity of the total column of $\mathrm{CO}_{2}(\mathrm{ppm})$ to the surface fluxes (top: based on the standard version 5A) and to the physics (bottom: using the CT surface fluxes). As expected, the fluxes control, to a large extent, the horizontal distribution of the $\mathrm{CO}_{2}$ concentrations and has a much larger impact on the $\mathrm{xCO}_{2}$ simulated values than the physics. The largest differences are located over poorly constrained regions (Siberia, Africa and Brazil) as it was the case for the surface fluxes. Compared to Figure 4 of the main article, the pattern of the differences remain unchanged in boreal summer, namely the negative anomaly over boreal latitudes. In boreal winter, the effect of the physics is strongly attenuated over Brazil and Africa as the CT fluxes are weaker than the CAMS fluxes over those regions. Indeed, the impact of the transport is all the more important that the surface fluxes are large.

The effect of the surface fluxes using physics 5A and the effect of the physics using the CT fluxes on the simulated $\mathrm{CO}_{2}$ mixing ratio have been quantified at surface sites at the annual and seasonal scales in Table 2 and Figure 4. Table 2 shows 

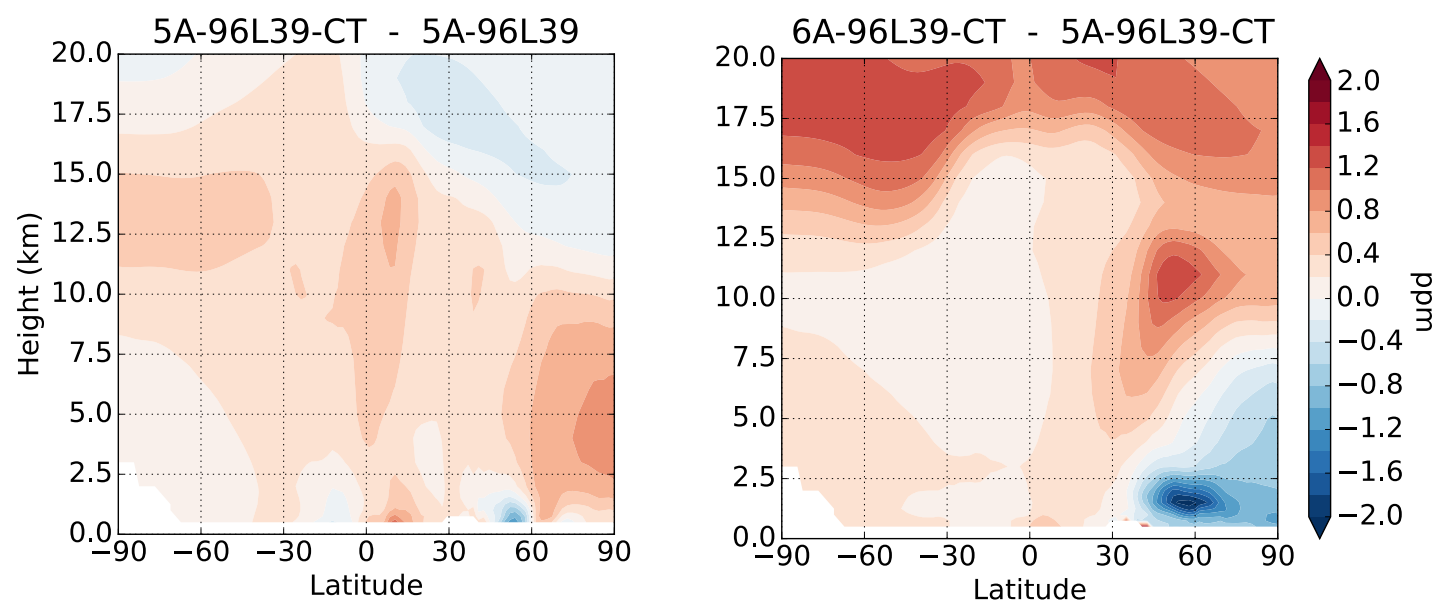

Figure 2. Left: Zonal-mean mixing ratio difference between 5A-96L39-CT and 5A-96L39 (effect of the fluxes). Right: Zonal-mean mixing ratio difference between 6A-96L39-CT and 5A-96L39-CT (effect of the physics) using CarbonTracker CT2017. The zonal mean is calculated for afternoon hours from 2005 to 2010 in summer (JJA). Corresponding to Figure 3 of the main paper.

the annual mean gradient to MLO on average over three latitudinal bands for the simulations described in Table 1. Based on this diagnostic, the physics has a negligible impact compared to the surface fluxes whose effect exceeds $1 \mathrm{ppm}$ in terms of differences between the northern and southern hemisphere.

\begin{tabular}{llll} 
VERSION & NORTHERN HEMISPHERE & TROPICS & SOUTHERN HEMISPHERE \\
\hline 5A-96L39 & $1.7(0.1)$ & $-0.4(0.1)$ & $-3.0(0.1)$ \\
6A-96L39 & $1.7(0.1)$ & $-0.4(0.1)$ & $-2.8(0.1)$ \\
5A-96L39-CT & $2.3(0.1)$ & $-0.4(0.1)$ & $-3.5(0.1)$ \\
6A-96L39-CT & $2.3(0.1)$ & $-0.3(0.1)$ & $-3.4(0.1)$
\end{tabular}

Table 2. Simulated mean gradient of the $\mathrm{CO}_{2}$ mixing ratios between MLO and other stations located in the Northern Hemisphere (latitudes $\left.>30^{\circ} \mathrm{N}\right)$, the Tropics $\left(30^{\circ} \mathrm{S} \leq\right.$ latitudes $\left.\leq 30^{\circ} \mathrm{N}\right)$, the Southern Hemisphere (latitudes $\left.<30^{\circ} \mathrm{S}\right)$. For each one of the three domains, the corresponding sites are weighted by the inverse of their standard deviation. The value inside the brackets defines the associated mean weighted standard deviation. Corresponding to Table 2 of the main paper.

At seasonal scale, Figure 4 shows that the change in surface fluxes has an impact opposite to that of the physics but similar 5 in terms of magnitude. The CT surface fluxes tend to decrease the seasonal amplitude. Here again, the sensitivity to the physics remains is similar with both CT fluxes and CAMS fluxes.

Like in the article, Figures 5, 6, and 7 evaluate the mean vertical profiles against independent airborne measurements over Northern America, Europe (EUR), Eastern Asia (EAS), Northern Southern Asia (NSA), India (IND) and Brazil. As above, the impact of the physical assumptions is small compared to the effect of the surface fluxes. For most of the seasons and the regions, 


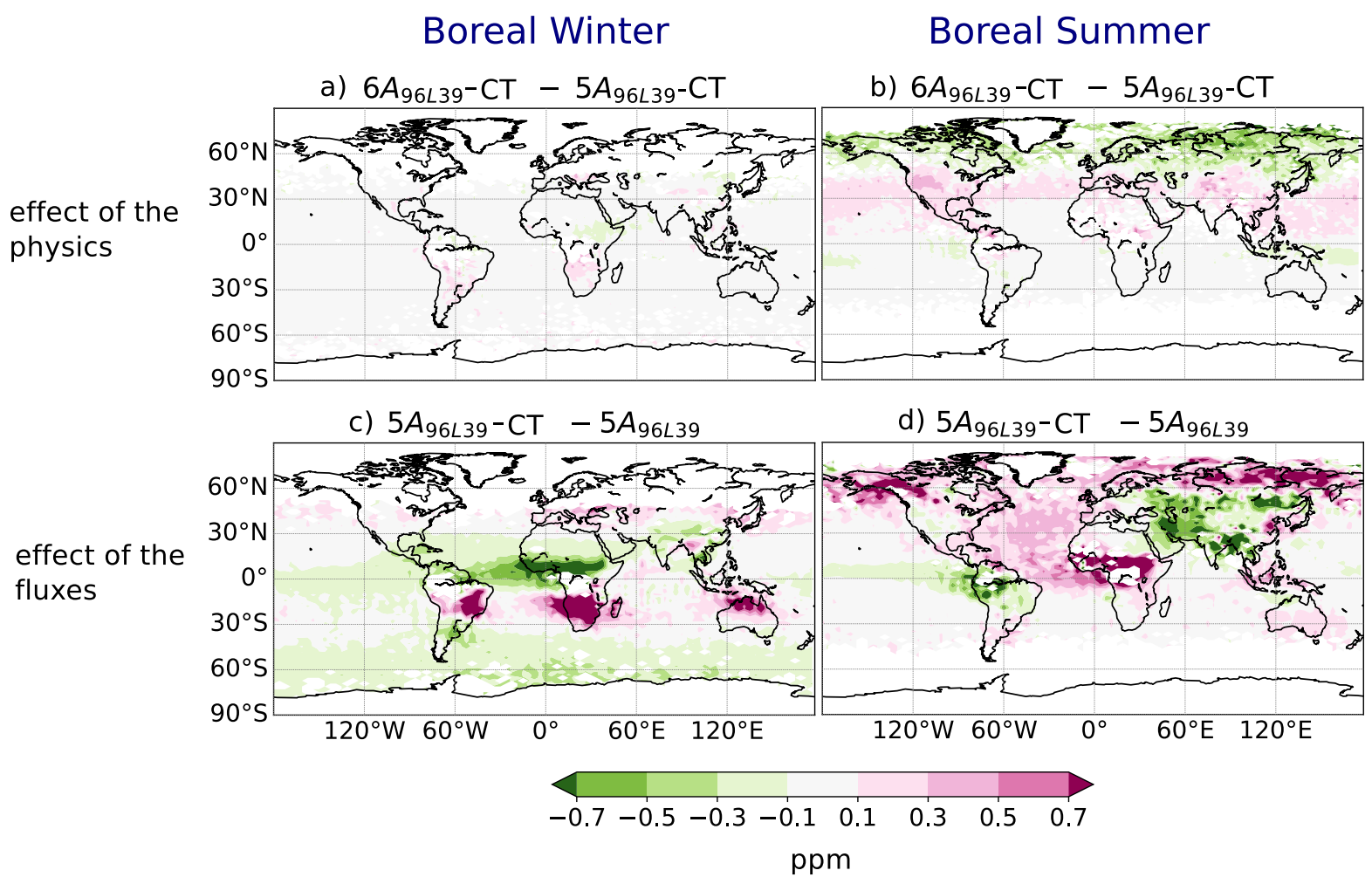

Figure 3. Map of the differences in $\mathrm{x} \mathrm{CO}_{2}$ (ppm) between 6A-96L39-CT and 5A-96L39-CT using CarbonTracker CT2017 (top, effect of the new physics) and 5A-96L39-CT and 5A-96L39 (last row, effect of surface fluxes). The left column shows the average over the 2005-2010 boreal winters (December-February) and the right column shows the average over 2005-2010 boreal summers (June-August). The simulated $\mathrm{x} \mathrm{CO}_{2}$ values have been temporally convolved with the sampling of the OCO-2 satellite retrievals for the year 2017. Corresponding to Figure 4 of the main paper.

the shape of the vertical profile is slightly modified for the $5 \mathrm{~A}$ and $6 \mathrm{~A}$ versions with the $\mathrm{CT}$ fluxes. The main conclusions about the effect of the physics still apply using the CT fluxes. The impact of the physics is overall small except over Brazil.

\section{Sensitivity to the surface fluxes: Conclusion}

We have performed 4 simulations: two simulations driven by the CAMS $\mathrm{CO}_{2}$ fluxes and two simulations driven by the CarbonTracker (CT) $\mathrm{CO}_{2}$ fluxes. For one given pair of simulations driven by either the CAMS or CT fluxes, the effect of a change of the physical assumptions or physics has been quantified on the simulated mixing ratios of $\mathrm{CO}_{2}$, including at locations and times of various measurement datasets. The findings can be summarized as follows:

1. The change of surface fluxes has a very strong impact on the $\mathrm{xCO}_{2}$ zonal mean distribution and on the mean shape of the $\mathrm{CO}_{2}$ vertical profile over India and Europe compared to the effect of the parameterization assumptions. The impact 

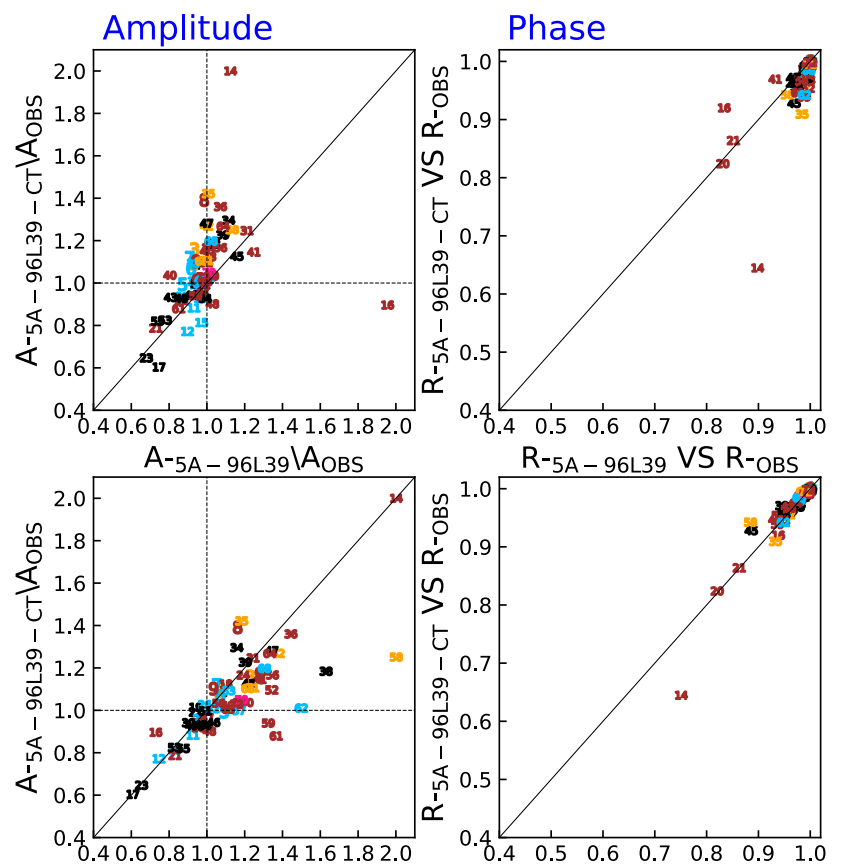

A- $6 \mathrm{~A}-96 \mathrm{~L} 39-\mathrm{CT} \backslash \mathrm{A}$ OBS

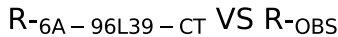

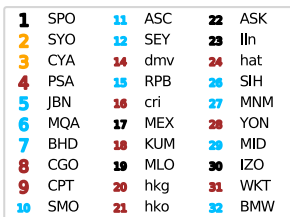

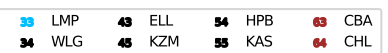

34 WLG 45 KZM 53 KAS $4 \mathrm{CHL}$

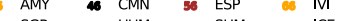

6 SGP 27 UUM 57 SHM 6 ICE

10 TTA STM

* UTA 0 PRS 50 NGL $\oplus$ PAL

THD

32 JUN 2 LUT

Figure 4. Top left: Ratio of the simulated to observed $\mathrm{CO}_{2}$ seasonal amplitude from 5A-96L39 (x axis) and 5A-96L39-CT (y axis) for all available stations. Top right: Correlations between the observed and simulated $\mathrm{CO}_{2}$ mean seasonal cycles from 5A-96L39-CT (y axis) and 5A-96L39-CT (x axis) for all available stations. Bottom: Same than top but from 5A-96L39-CT (y axis) and 6A-96L39-CT (x axis). The stations are numbered by increasing latitude (with the identifier correspondence given in the bottom of the panel) and are colored according to their category. Blue: maritime stations, black: mountainous stations, yellow: coastal station, brown: continental station. Stations written in lowercase (uppercase) refer to unassimilated (assimilated) stations. Corresponding to Figure 6 of the main paper

is more modest and of similar magnitude than the impact of the physics at monitoring surface sites and over NSA, EAS and over Northern America.

2. Regarding the impact of the physics, the findings of the article still apply with the CT fluxes. The convective inhibition decreases the mean values of $\mathrm{xCO}_{2}$ over the boreal latitudes in summer and increases the amplitude of the seasonal cycles at surface sites. Regarding the evaluation of the simulations against independent airborne measurements, the impact of the physics remain small except over Brazil and northern America in summer (greater than 1 ppm). 


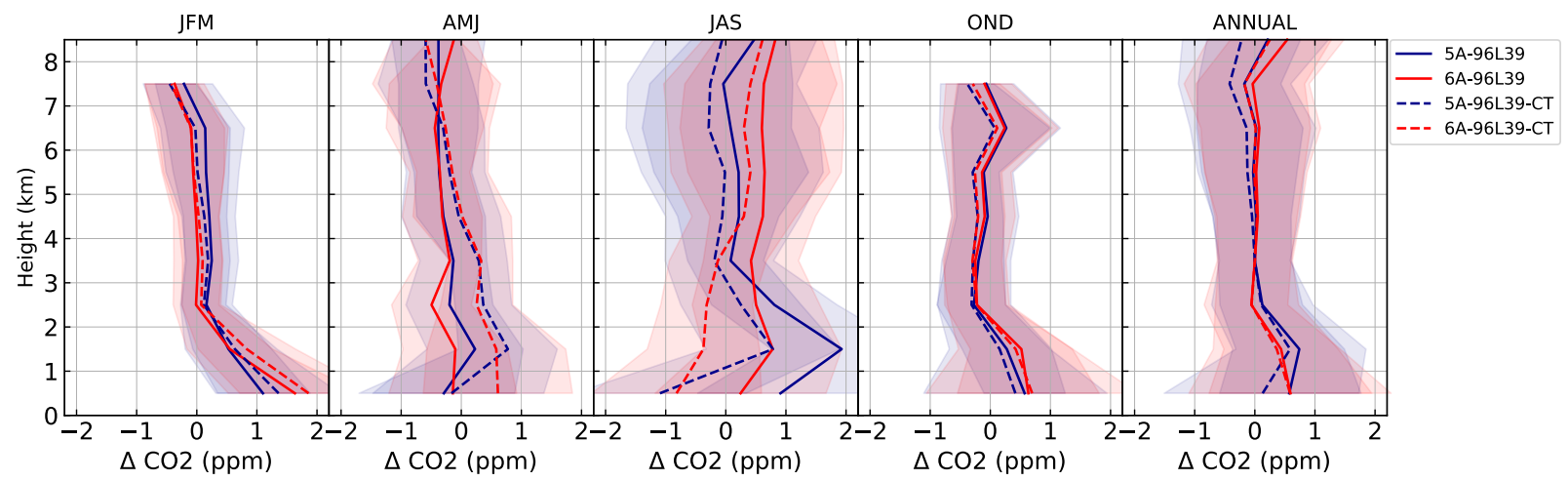

Figure 5. Bias (model-observations, thick lines) and standard deviation (shaded areas) for the monthly $\mathrm{CO}_{2}$ vertical profile misfits over North America during the period 2008-2014. The data have first been averaged in 1-km-altitude-bins per hour and per site, before being averaged among the 12 North American sites of Figure 2 per month. The statistics are drawn from that ensemble of monthly and spatiallyaveraged values. They are shown for each season (January-March, JFM; April-June, AMJ; July-September, JAS; October-December, OND) and for the whole year. In order to highlight the differences in profile shape, the annual mean of the bias at $3.5 \mathrm{~km}$ has been removed for each simulated vertical profile (5A-96L39: -2.0 ppm, 6A-96L39: -2.0 ppm, 5A-96L39-CT: 1 ppm and 6A-96L39-CT: -1.2 ppm). Corresponding to Figure 10 of the main paper. 

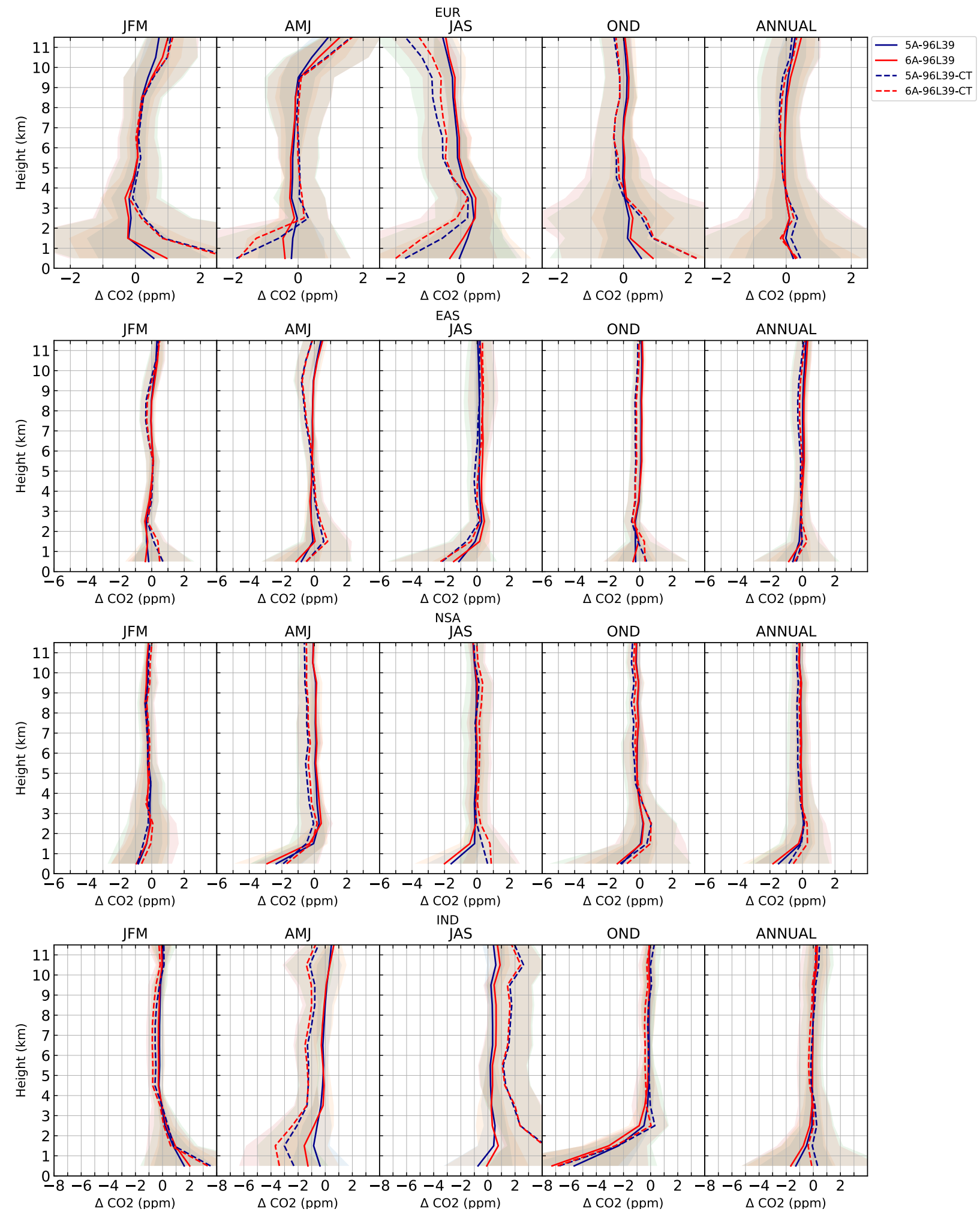

Figure 6. Same as for Figure 5 but over Europe (EUR), Eastern Asia (EAS), Northern Southern Asia (NSA) and India (IND) from the CONTRAIL dataset during the period 2006-2011. The domain is portraited in Figure 2 of the main article. Corresponding to Figure 11 and 12 of the main paper. 

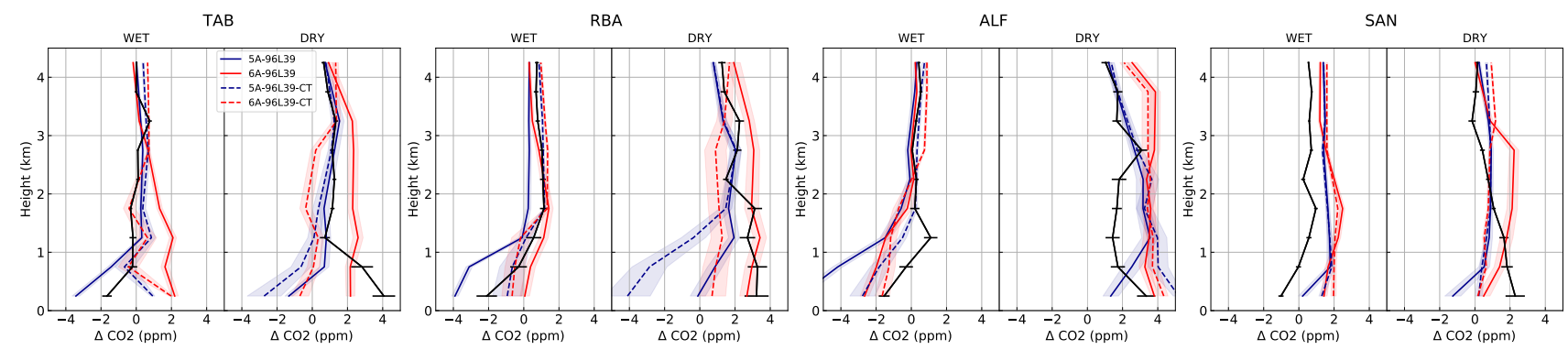

Figure 7. Mean difference between $\mathrm{CO}_{2}$ profiles measured and simulated in 2010 at the four Amazonian aircraft sampling sites and an oceanic $\mathrm{CO}_{2}$ background (that is, $\mathrm{\Delta C \textrm {O } _ { 2 }}$ ) during the dry (left of each panel) and wet (right of each panel) seasons, respectively (solid lines) and the standard deviation divided by the square root of number of profiles (dashed lines and error bars). The background is estimated from in situ measurements at monitoring stations ASC and RPB, as described in the main text. The dry season (red lines) is affected by fires at most sites and is here defined as period July-October for illustrative purposes only; it does not correspond to all months within the fire season. Corresponding to Figure 13 of the main paper. 